Relations industrielles

Industrial Relations

\title{
Le remplacement du personnel
}

\section{Germain Giroux}

Volume 3, numéro 5, janvier 1948

URI : https://id.erudit.org/iderudit/1023591ar

DOI : https://doi.org/10.7202/1023591ar

Aller au sommaire du numéro

\section{Éditeur(s)}

Département des relations industrielles de l’Université Laval

\section{ISSN}

0034-379X (imprimé)

1703-8138 (numérique)

Découvrir la revue

Citer cet article

Giroux, G. (1948). Le remplacement du personnel. Relations industrielles /

Industrial Relations, 3(5), 69-71. https://doi.org/10.7202/1023591ar

Tous droits réservés @ Département des relations industrielles de l’Université Laval, 1948
Ce document est protégé par la loi sur le droit d'auteur. L’utilisation des services d'Érudit (y compris la reproduction) est assujettie à sa politique d'utilisation que vous pouvez consulter en ligne.

https://apropos.erudit.org/fr/usagers/politique-dutilisation/ 
Il est indéniable que là où plusieurs peinent en commun, y apportant le travail et le capital, il doit y avoir une autorité. Autrement, on courrait au désastre et au désordre. Mais en vertu de quel droit et de quel principe cette autorité directrice serait-elle toujours et uniquement aux mains des propriétaires?

\section{Lutte des classes}

C'est là le point névralgique de la lutte entre le capital et le travail; c'est parce que la propriété a pris, à elle toute seule, le manteau de l'autorité, qu'elle est aujourd'hui battue en brèche; c'est pour cela aussi que s'est établi le présent ré: gime de salariat, régime qui fait que le travail, et indirectement la personne même de l'ouvrier, demeure une marchandise soumise nécessairement à la loi de l'offre et de la demande, du beau et du laid, du fort et du faible, du vieux et du jeune, du lent et du vif, du vigoureux et de l'anémique, et ainsi de suite, selon les goûts et les besoins.

La société coopérative pose le même problème si les exécutants sont autres que les membres de la coopérative.
La société par actions pose aussi le même problème, à cette différence près que, souvent le capital étant anonyme, le contrôle s'exerce par quelques individus dont la puissance est considérable et peut s'étendre, en définitive, à une foule d'entreprises diverses. C'est ainsi, par exemple, que par le mécanisme de la loi quelques individus qui dirigent l'exploitation d'une mine d'amiante, peuvent en venir à contrôler toutes les manufactures qui font subir des transformations à ce minérai et les magasins qui en font la vente, établissant ainsi le cycle complet du monople.

La propriété de l'Etat, à son tour, peut engendrer les mêmes résultats, parce que le contrôle s'étend à toute lactivité nationale et peut permettre, comme dans les dictatures, à un seul homme de dicter à son gré l'économie totale de la nation.

\section{Conclusion}

L'on voit par ce tableau que l'entreprise moderne, d'avance grevée de servitudes sociales, servitudes qui naissent de sa constitution même et des relations yu'elle doit nécessairement avoir avec le reste de la collectivité, dépasse la notion véritable et traditionnelle du régime propriété.

\section{LE REMPLACEMENT DU PERSONNEL}

\section{Germain GIROUX}

Le remplacement du personnel cause bien des soucis aux patrons. Toute entreprise, désireuse de lutter avantageusement sur le marché, doit pouvoir compter sur une main-d'oeuvre experte et stable, et il n'est pas toujours loisible au patron d'embaucher de nouveaux employés qualifiés; d'où la nécessité pour l'industrie d'entraîner ses nouveaux employés, de former ses apprentis et de pourvoir aux frais de leur entraînement. Quand on sait que le coût de la formation d'un apprenti se chiffre par centaines de dollars, par milliers quelquefois, il va de soi qu'un patron ne puisse laisser partir un ouvrier expérimenté sous un prétexte futile, car alors ce sera une entreprise rivale qui profitera des dollars dépensés par le pemier. Chaque fois que l'employeur perd un ouvrier experte, c'est une certaine somme d'argent qui part avec cet ouvrier, sans aucun espoir de retour. Il y a encore plus.

Il ne faut pas quine entreprise ait la réputation d'être une bonne école de formation mais, d'autre part incapable de garder sa main-d'oeuvre expérimentée. La renommée d'un produit est en partie le résultat de l'expérience de la main-d'oeuvre qui l'a fabriqué; il en résulte que la perte d'ouvriers expérimentés entraîne une diminution dans les profits justement prévus.

En conséquence, il est nécessaire de réduirc à son strict minimum le pourcentage de remplacement-du personnel. On objectera peut-être que la chose n'est pas facile, que l'ouvrier est instable ou qu'il est impossible d'adapter à notre milieu les systèmes appliqués sur une haute échelle aux Etats-Unis. Pourtant une des entreprises les plus importantes du Québec, grâce à l'esprit d'initiative de ses propriétaires, a réussi depuis deux ans, à mettre en valeur chez elle ce genre de relations industrielles, si populaire chez nos voisins du Sud. La Compagnie Limitée Dominion Corset, la plus importante du genre dans tout l'Empire, peut aujourd'hui être fière du succès obtenu par l'appli- 
cation de méthodes rationelles et humaines aux conditions de travail.

Il y a quelques années, le taux de virement du personnel, variait de $12 \%$ à $18 \%$, et aujourd'hui il n'est que de $0.8 \%$ à $4 \%$. Comment un tel succès a-t-il été obtenu? Voyons ...

Les causes du départ d'un employé sont parfois incontrôlables. Cependant, tout patron peut et doit éviter les départs, qui résultent de la mésentente, de la mauvaise santé, du traitement, du mécontentement ou de l'incompréhension . . . Dominion Corset s'est attaquée à ces divers problèmes, en cherchant à éliminer, si possible le mal à sa racine.

L'entreprise ne considère pas l'ouvrier comme une machine qu'il faut conrtaidre au plein rendement, mais comme un être humain raisonnable et sensible.

Par une série de services industriels, la Compagnie se préoccupe d'abord de satisfaire les légitimes besoins de ses employés. C'est ainsi que la médecine industrielle fut introduite chez nous.

A la Dominion Corset, le personnel est en majorité féminin, donc plus délicat à diriger, si la méthode n'est pas adéquate.

Avant l'embauchage, tout aspirant doit subir des tests d'aptitude et des tests spsychologiques, afin d'établir ses qualifications. Après quoi il doit se soumettre à un examen médical complet, comprenant :

a) L'examen de la vue au moyen du Keystone Survey Test en vue de déterminer l'état de la vision, et du Dvorine Test, pour la perception des couleurs.

b) L'examen dentaire afin de déceler non seulement les caries, mais aussi toute malformation ou défectuosité buccale, pouvant entraîner des troubles de gorge ou d'estomac.

c) L'examen médical proprement dit :

Radiographie des poumons et analyse du sang, afin de relever les traces de tuberculose ou de maladies vénériennes; cet examen proprement médical terniné, le médecin fait du sujet une approche psychologique et psycho-somatique. En effet, on ne peut oublier que l'ouvrier apporte à son travail toutes ses tendances naturelles et que, livré à ses seules ressources, il éprouvera souvent de la difficulté à équilibrer ses problèmes psychologiques élémentaires ou complexes.

Par ces approches, on note ses états cardiaques, ses réactions émotives et tout ce qui pourrait modifier son équilibre intérieur, au cours de la période d'emploi.
L'enquête se termine par un questionnaire sur les habitudes alimentaires de l'aspirant. Tout le monde sait qu'il faut se nourrir, mais bien peu savent se bien nourrir. Ici, on conseille à l'aspirant une diète appropriée et on lui inculque les principes de lhygiène industrielle en même temps que les soins corporels à se donner.

A chacune de ces étapes, une fiche médicale est remplie, non pas tant pour renseigner la Compagnie sur l'état général de l'aspirant que pour aider ce dernier après son embauchage. L'aspirant étant déclaré physiquement apte, ses futurs compagnons de travail l'accepteront amicalement, car ils n'auront pas à craindre un danger quelconque de contamination. Lors de l'entrée en fonction, on applique au nouvel employé les règlements, les conditions de travail et de salaire, les chances d'avancement, puis on le présente à qui de droit, car il est nécessaire que l'arrivant se sente chez lui dès les premiers instants. D'où une première satisfaction pour l'apprenti de se savoir considéré comme un être humain et non comme une chose perdue dans un grand tout.

L'apprenti fait un premier stage à un centre d'entraînement, où il a l'occasion de s'initier à tous les secrets de son nouveau métier sous la surveillance de compagnons experts. L'ouvrier aura déjà remarqué l'éclairage moderne des départements, complété par un ensemble de couleurs pastel. Avant que la première journée soit terminée, d'autres agréables surprises l'attendent: repos de 10 minutes le matin et l'après-midi avec distribution gratuite de lait à tous; conditions d'hygiène industrielle impeccables; grande propreté de l'atelier et esprit d'entr'aide. S'il dîne à l'atelier, une salle l'attend où on lui sert des soupes, des breuvages et des fruits gratuitement, tout en lui permettant d'écouter son programme radiophonique favori, à l'heure du lunch. Un accident ou un malaise survient-il au cours de son travail, une gardemalade est à sa disposition, et il peut consulter, en tout temps et gratuitement, le médecin de la Compagnie, qui passe tous les avant-midis à l'établissement. Les médicaments de première nécessité sont fournis à titre gratuit, et si des remèdes, des fortifiants ou extraits glandulaires sont nécessaires, la Compagnie les achète pour l'employé, qui rembourse par versements hebdomadaires minimes. Il en est ainsi pour les soins dentaires, offerts par nos deux dentistes, à taux fixes, payables par la Compagnie et remboursables par l'employé à raison de $\$ 2.00$ par semaine. Tout examen, médical, dentaire ou de la vue, se fait aux frais exclusifs du patron, ainsi que la consultation. Mais les services ne s'arrêtent pas là. 
Chaque employé a pleine liberté de formuler ses griefs à la direction, d'exposer ses plaintes et de demander aide et conseil pour toute question d'ordre personnel, familial, social ou autre. Tous et chacun sont traités sur un pied d'égalité avec justice et courtoisie. Les employés ont ainsi l'impression de faire partie d'une grande famille où il fait bon travailler, car une discipline «familiale » règne dans notre entreprise de sorte que les patrons sont heureux de protéger ainsi la santé physique et morale des futures mamans de notre race.

On pourrait aussi parler des organisations sportives et sociales qu'encourage la Compagnie, mais passons pour l'instant.

On demandera quel résultat tout ce système a produit et quel peut être le coût approximatif de ces services?

Nous ne craignons pas d'affirmer que le taux de virement de notre personnel est l'un des plus bas du Québec, puisqu'il varie seulement de $0.8 \%$ à $4 \%$ avec une moyenne de $1.5 \%$, d'où économie dans le coût de formation et d'entraînement. En second lieu, les taux d'absence sont de $2.7 \%$ pour les absences contrôlables, avec une différence de seulement $1 \%$ pour les absences incontrôlables, ce qui est un record dans la grande industrie du Québec. Un troisième résultat acquis : la santé de nos ouvriers a atteint un niveau supérieur à la moyenne industrielle, et chez nous, c'est l'exception qui est affligée d'un « teint d'industrie et de factorie ». Un quatrième résultat, découlant des pre- miers : la stabilité du personnel expert, qui facilite le gain de meilleurs salaires sans l'exigence d'un rendement accru. Et la chose s'explique facilement quand on sait que l'ouvrier travaille dans un milieu invitant et propre, où il est tenu compte de tout facteur humain et de la personnalité de chacun.

Pour une entreprise qui emploie près de mille personnes, il va sans dire que l'organisation et le maintien de tels services nécessitent une dépense de quelques milliers de dollars, soit une moyenne annuelle de $\$ 15$. par employé, pour les services médicaux seulement. Le secret d'une telle réussite réside dans une franche collaboration entre la direction et le médecin, basée sur une éducation progressive du personnel.

Le responsable de cette politique vis-à-vis la direction de la Compagnie est le Gérant du Personnel. Il a son propre budget à administrer et à lui échoit le rôle de maintenir une juste harmonie dans l'industrie. Dans la petite entreprise, cette responsabilité sera cumulée avec celle des ventes, des achats, de la finance ou de la gérance générale.

Si une de nos entreprises a réussi à abaisser le taux de remplacement du personnel à son strict minimum par l'organisation de services médicaux, industriels et sociaux, pourquoi d'autres ne suivraient-elles pas la même voie? Le succès de l'industrie est à ce prix sans compter qu'un tel procédé sert l'entente entre les classes de la société.

\section{PERSONNEL MENAGEMENT}

\section{Germain GIROUX}

The question of staff replacement causes employers a great deal of concern. In order to keep going, let alone progress, any enterprise has to be able to count an expert and steady working force, and it is not always convenient for the employer to hire new employees that are qualifield; hence the need for a given industry to train its own new employees, to school apprentices and stand the expense of their training. Considering that the cost of training an apprentice usually figures up in the hundreds, sometimes in the thousands, of dollars, it goes without saying that no employer can afford to let out an experienced workman for any paltry reason; for that would only be to see some rival enterprise profit from the money expended by oneself. Every time an employer loses an expert workman, it represents a certain sum of money gone, along with the workman, without any hope of return. But there is something else besides.

An enterprise does itself no particular good by developing a reputation as a good training school, if on the other hand it is incapable of holding its experienced help. The reputation of a product is partly the result of the experience of the workers employed in its production; from this it follows that the loss of experienced workers causes a decrease in the profit the enterprise is entitled to expect. 